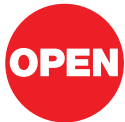

SUBJECT AREAS:

COSMOLOGY

COMPUTATIONAL ASTROPHYSICS

PHYSICS

FLUID DYNAMICS

Received

11 January 2012

Accepted

1 May 2012

Published

4 December 2012

Correspondence and requests for materials should be addressed to

A.M. (amitra@barc. gov.in)

\section{Interpretational conflicts between the static and non-static forms of the de Sitter} metric

\author{
Abhas Mitra \\ Homi Bhabha National Institute, Mumbai, India.
}

The de-Sitter metric is a special form of the non-static Friedmann metric, and appears to be genuinely non-static since it describes the initial exponential expansion of the Big Bang universe. However, the de Sitter metric appears to be perfectly static in the Schwarzschild frame where the vacuum fluid is supposed to be in motion. Here we highlight the conflicts between the static and non-static versions of the de-Sitter metric from a physical perspective. In particular, while the "Principle of Energy Conservation" is honored in one case, the same is badly violated for the other. However, we offer a partial resolution of such conflicts by deriving the static de Sitter metric by solving the relevant field equations. It is seen that, it is the very special vacuum equation of state pressure $=-$ density which results in the static form even when the vacuum fluid is supposed to be in motion.

$\mathrm{n}$ general relativity, in principle, one is free to use arbitrary coordinates. Indeed for studying a spherically symmetric fluid, one uses both the comoving coordinates, $(r, t)$, as well as the curvature or Schwarzschild coordinates $(R, T)$; and the angular coordinates remain the same in both the cases. In the comoving frame, by definition, a fluid element is at a fixed radial coordinate $r$ and local 3-speed of the fluid $v(r, t)=0$. Though the comoving coordinates stick to the fluid, the expansion or contraction of the fluid can be studied by considering the motion of nearby fluid elements. In fact one can define a volume expansion scalar as the covariant divergence of the fluid 4-velocity field $u^{i 1,2}$ :

$$
\Theta=u^{i} ; i
$$

to quantify the expansion/contraction in an invariant manner. Physically, the expansion scalar measures the fractional rate at which the volume of a small ball of matter changes with respect to time as measured by a comoving observer lying at the centre of the ball. On the other hand, by definition, in a non-comoving frame, for a non-stationary case, the fluid element is in motion even with respect to an observer whose position momentarily coincides with a certain fluid element. For instance, for a non-stationary situation, an observer sitting at a given radial coordinate $R$ finds the fluid to be flowing past it with a local 3-speed ${ }^{3}$ :

$$
v(R, T)=\frac{e^{\lambda / 2} d R}{e^{\eta / 2} d T}
$$

where the Schwarzschild frame is described by the metric

$$
d s^{2}=e^{\eta(R, T)} d T^{2}-e^{\lambda(R, T)} d R^{2}-R^{2} d \Omega^{2}, \quad d \Omega^{2}=\left(d \theta^{2}+\sin ^{2} \theta d \phi^{2}\right)
$$

Incidentally, this Schwarzschild radial coordinate $R$ is also called the "area coordinate" as $4 \pi R^{2}$ represents the invariant area of symmetric 2 -spheres around the centre of symmetry. Thus the Schwarzschild radial coordinate is actually a scalar/invariant quantity with an inherent spacelike character. This is so because, by initial definition, the centre of symmetry corresponds to a point at $R=0$ possessing an invariant surface area $4 \pi R^{2}=0$. Since a noncomoving observer explicitly finds the fluid to be in motion unlike the comoving observer, the temporal dependence of the pertinent physical quantities are expected to be more pronounced or complex. To appreciate this consider a perfect fluid in its comoving frame in which the components of the matter energy momentum tensor are $(G=c=1)$ :

$$
T_{0}^{0}(\operatorname{com})=\rho_{e}(r, t) ; \quad T_{1}^{1}(\mathrm{com})=T_{2}^{2}(\mathrm{com})=T_{3}^{3}(\operatorname{com})=-p_{e}(r, t)
$$

and all other components of $T_{b}^{a}$ are zero. Here 


$$
\rho_{e}(r, t)=\rho(r, t)+\Lambda / 8 \pi
$$

is the effective matter energy density and

$$
p_{e}(r, t)=p(r, t)-\Lambda / 8 \pi
$$

is the effective isotropic pressure when cosmological constant $\Lambda$ is present. But the expressions for the components of the energy momentum tensor in the Schwarzschild frame, obtained via a local Lorentz transformation, are far more complex ${ }^{3}$ :

$$
\begin{gathered}
T_{0}^{0}(\text { ncom })=\frac{\rho_{e}+p_{e} v^{2}}{1-v^{2}} \\
T_{1}^{1}(\text { ncom })=T_{2}^{2}(\text { ncom })=T_{3}^{3}(\text { ncom })=-\frac{p_{e}+\rho_{e} v^{2}}{1-v^{2}}, \\
T_{0}^{1}(\text { ncom })=\frac{\left(p_{e}+\rho_{e}\right) v e^{(\eta-\lambda) / 2}}{1-v^{2}},
\end{gathered}
$$

and $T_{0}^{2}($ ncom $)=T_{0}^{3}($ ncom $)=0$. Therefore, the solutions of the Einstein equation

$$
G_{k}^{i}=-8 \pi T_{k}^{i},
$$

must be far more complex in the non-comoving case because $T_{k}^{i}\left(\right.$ ncom) (Eqs.[7-9]) is far more complex than $T_{k}^{i}(\mathrm{com})$ (Eq.[4]). Naturally, in view of such complex nature of the energy momentum tensor and explicit motion of the fluid, the non-comoving solutions are expected to have more pronounced temporal dependence than their comoving counterparts. And thus non-stationary GR problems such as gravitational collapse or cosmology become mathematically more tractable in terms of the comoving coordinates $r, t$ than in terms of $R, T$.

Here it may be helpful to briefly remind the reader about the definitions of "Stationary" and "Static Metric". If there is a coordinate system for which none of the metric coefficients contain any temporal dependence, then the metric is called "Stationary"4. A familiar example is the Kerr metric, and one expects the spactime represented by the Kerr metric to be stationary irrespective of any coordinate transformations it might be subjected to. And a static metric is a sub-class of stationary metrics where there are, in addition, no spacetime cross-terms $d x^{0} d x^{\alpha 4}$. Thus when there is no spacetime cross term, the two terms "non-stationary" and "non-static" become synonymous. The most familiar spherically symmetric static metric is the vacuum Schwarzschild metric:

$$
d s^{2}=(1-2 M / R) d T^{2}-(1-2 M / R)^{-1} d R^{2}-R^{2} d \Omega^{2}
$$

On the other hand, metrics associated with gravitational collapse or evolving cosmology naturally correspond to non-stationary or nonstatic ones though, of course, the respective final states, by definition, should correspond to static metrics. The best example of such a nonstatic metric is the Friedmann Robertson Walker (FRW) metric which is the cornerstone of modern cosmology:

$$
d s^{2}=d t^{2}-a^{2}(t)\left[\frac{d r^{2}}{1-k r^{2}}+r^{2} d \Omega^{2}\right]
$$

where $k=+1,0,-1$ and $a(t)$ is the scale factor. As is well known, this form of the FRW metric corresponds to the comoving frame in which the cosmic fluid is at rest $\left(v_{c o m}=0\right)^{1,2}$. The metric is still non-static because

$$
g_{r r}=g_{r r}(t)=\frac{a^{2}(t)}{1-k r^{2}}
$$

However the form of the metric is still simple in the sense that $g_{00}=1$ and $g_{r r}(t)$ has a rather benign form. Though in principle, all comoving metrics must be expressible in the Schwarzschild form, the fact is that, even for this simple metric, a general Schwarzschild form is still not known! The difficulty in having a Schwarzschild form of the simple comoving metric, may be attributed to the complex form of $T_{k}^{i}($ ncom); i.e., Eqs.(7-9).

As already mentioned, there could be an invariant measure of the volume expansion of the FRW fluid in terms of the volume expansion scalar; and for the FRW metric, one finds ${ }^{1,2}$

$$
\Theta=3 \frac{\dot{a}}{a}
$$

where an overdot denotes differentiation by $t$. Clearly, the cosmic fluid can be static in an invariant manner only if one would have a static metric with $\dot{a} / a=0$; i.e., for Einstein's static universe.

Now we consider a very special case of the FRW metric, namely the de Sitter Metric:

$$
d s^{2}=d t^{2}-e^{2 H t}\left(d r^{2}+r^{2} d \Omega^{2}\right)
$$

In particular, for this FRW metric, one has

$$
a(t)=e^{H t} ; \quad k=0
$$

where the "Hubble Parameter"

$$
H=\frac{\dot{a}}{a}=\sqrt{\Lambda / 3}
$$

It would be interesting to compare the de Sitter solution with another well known FRW solution, namely, the Einstein de Sitter dust solution ${ }^{1,2}$ :

$$
a(t) \propto t^{2 / 3} ; \quad k=0
$$

or the solution for the radiation dominated era $a(t) \propto t^{1 / 2}$.

Clearly, the temporal dependence of the de Sitter metric is far more extreme as compared to the Einstein de Sitter metric or many other FRW solutions. In particular, for the solution (18), the volume within the particle horizon increases as $V_{p h} \sim t^{3}{ }^{1,2}$. Even with this fast increase of volume, at the epoch of de-coupling of matter and radiation, it turns out that $V_{p h}^{d}$ is only a tiny fraction $2.10^{-5}$ of the volume of the causally connected region. Then question arises, how could the cosmic microwave background be so homogeneous despite the lack of any causal connection amongst distant patches of the initial universe. This problem is known as the "Horizon Problem"; and as is well known, is remedied by postulating an exponentially expanding "inflationary phase" immediately after the "Big Bang"1,2. The volume of the universe is supposed to have blown up by a factor of $\sim 10^{78}$ during this flash lasting between $\sim 10^{-36}-10^{-32} \mathrm{~s}$. The "flatness" puzzle of the present universe is similarly removed by postulating the same initial exponential inflationary phase. Clearly, any expectation that the de Sitter phase should be intrinsically static in view of $a$ fixed vacuum energy density, $\rho_{e}=\Lambda / 8 \pi$, is not honored here.

Indeed, the invariant non-static nature of the inflationary or the de Sitter phase can be gauged by finding the corresponding volume expansion scalar:

$$
\Theta=3 \frac{\dot{a}}{a}=3 H=\sqrt{3 \Lambda}
$$

It is clear that this inflationary expansion can appear to be invariantly nonexistent only if one would have $\Lambda=0$. Further as already explained, for a non-comoving case, the solutions are expected to be more complex with likely more pronounced temporal dependence. However, in a very surprising manner, this appears not to be the case for the corresponding Schwarzschild observer:

The Schwarzschild observer does not at all perceive the temporal variation of the scenario. This is so because, the Schwarzschild form of this very special FRW metric is known, and it is a static one $e^{5,6}$

$$
d s^{2}=\left(1-\frac{\Lambda R^{2}}{3}\right) d T^{2}-\left(1-\frac{\Lambda R^{2}}{3}\right)^{-1} d R^{2}-R^{2} d \Omega^{2}
$$

and which may be also written as

$$
d s^{2}=\left(1-H^{2} R^{2}\right) d T^{2}-\left(1-H^{2} R^{2}\right)^{-1} d R^{2}-R^{2} d \Omega^{2}
$$


To summarize, in the comoving frame where the fluid is at rest, $v_{\text {com }}$ $=0$, the de Sitter solution has an extremely rapid temporal variation: $a(t) \sim e^{H t}$. On the other hand, in the non-comoving Schwarzschild frame, where one would normally expect a more complex temporal dependence, the metric is static! This static Schwarzschild form is surprising for other reasons too:

Though it is an interior solution for the dark vacuum fluid, it looks like the exterior vacuum Schwarzschild solution. To explore this similarity further, consider a spherical fluid of uniform density $\rho_{0}$ and having a radius $R_{b}$. The gravitational mass of this fluid is

$$
M=\frac{4 \pi}{3} \rho_{0} R_{b}^{3}
$$

so that, at the exterior vacuum boundary, $R=R_{b}$, the corresponding Schwarzschild metric becomes

$$
d s^{2}=\left(1-A^{2} R^{2}\right) d T^{2}-\left(1-A^{2} R^{2}\right)^{-1} d R^{2}-R^{2} d \Omega^{2}
$$

where

$$
A^{2}=\frac{8 \pi}{3} \rho_{0}
$$

Thus the exterior vacuum solution at $R=R_{b}$ (23) would look exactly similar to the interior de Sitter solution (21).

Recall that when de Sitter presented his metric in 1917, it was only in this static form $(20)^{7}$; and this was preceded by Einstein's static universe:

$$
d s^{2}=d T^{2}-\left(1-\Lambda R^{2}\right)^{-1} d R^{2}-R^{2} d \Omega^{2}
$$

where both the dark fluid $(\Lambda)$ and matter are at rest and strictly no red/blue shift of "nebulae" is to be expected. Note de Sitter discovered his static metric by intutitively extending this metric for Einstein's static universe $\mathrm{s}^{5}$ on geometrical considerations and not by solving any field equations in an independent manner.

In the de Sitter case, there was no matter and the vacuum fluid appeared to be at rest. Indeed Tolman derived (20) by assuming $v=$ $0^{6}$. Yet in order to explain the actual universe having "nebulae", it was thought that the matter density was neglible in comparison to vacuum density $\Lambda / 8 \pi$. The movement of an odd galaxy was considered to be the motion of a test particle with negligible energy density in the background of the static vacuum fluid ${ }^{5}$.

On the other hand, non-static fleld equations were invoked for the first time by Alexander Friedmann in 1921. And later, as the FRW metric got derived in a comprehensive manner, primarily on strong symmetry arguments, most of the cosmologists started believing that the universe was indeed expanding, and did not spare much attention to either Einstein's or de Sitter's static metrics. On the other hand, the comoving form of the de Sitter metric, as a special case of the FRW metric, became familiar to all cosmologists. Though some books do mention about the original static de Sitter metric, many of the present day cosmologists or particle physicists many not be even aware that non-static de Sitter metric studied by them had originally appeared in a static form. And even when they may be recalling the original static form of the de Sitter universe, their interest may remain focused on geometrical peculiarities like the de Sitter hyperboloid can be embedded in a flat 5-D Minkowski spacetime ${ }^{1}$.

But, we would like to highlight that, clearly, there is a physical or at least interpretational self-contradiction between the original de Sitter view and the present day non-static de Sitter view:

Suppose, one says that in view of the constant energy density $\rho_{e}=$ $\Lambda / 8 \pi$, of the vacuum fluid, it is natural that de Sitter metric must be static. In fact this was the original de Sitter view (that time, there was hardly any notion of a non-stationary metric). In this original view, the vacuum is static, $v(R, T)=0$, though a test particle with negligible mass can move in this static background just like a test particle can move around a static star described by the exterior vacuum Schwarzschild metric (11). So in the original de Sitter universe, one has

$$
\rho_{\text {matter }}=0 ; \quad v(R, T)=0 \text { for vacuum fluid }
$$

If one would like to explain this inherent staticity due to constancy of vacuum energy density, then one should not expect the vacuum fluid to be expanding in any frame, because, physically any expansion should lead to a decrease of energy density.

However, as GR grew and non-stationary scenarios were encountered, the notion of "stationary" got linked not to the constancy or non-constancy of energy density. On the other hand, as already mentioned, a "stationary" metric would be one where none of the coefficients have any temporal dependence. Further, once one would find a metric to be "stationary", one would expect to remain so even after a physically significant coordinate transformation. This is so because, after all, a metric represents a physical view point and by principle of covariance, the essential physical picture should not depend on the choice of coordinates. For instance, as already mentioned, the vacuum Schwarzschild metric is supposed to represent an inherently static physical picture irrespective of the coordinates used. But as the focus shifted on the comoving version of the de Sitter metric, it was considered to be actually non-static even though it corresponds to a fixed energy density.

Obviously one cannot subscribe to both these views that the de Sitter universe is both, naturally, "static" and "non-static" simply because they are self-contradictory.

Further, after the development of the notion of the non-stationary spacetime, the present day view is that in the non-comoving Schwarzschild frame, the vacuum fluid is moving with a 3-speed

$$
v(R, T)=\left(1-H^{2} R^{2}\right)^{-1} \frac{d R}{d T}
$$

even though the corresponding metric (20) is static. This view gets supported by the fact that $\Theta=\sqrt{3 \Lambda} \neq 0$ if $\Lambda \neq 0$.

And of course, in the FRW picture galaxies are not mass-less test particles, but, on the other hand they form the cosmic fluid. In this view, the space; i.e., the vacuum expands and the galaxies just remain embedded in this expanding space and partake of the expansion. Further for the present $\Lambda \mathrm{CDM}$ cosmology, in the present epoch,

$$
\rho_{\text {matter }} \sim \rho_{\text {vac }}
$$

Such glaring contradictions between the two versions of the de Sitter picture, to the best knowledge of this author, have never been brought out. And obviously there have not been any attempts for physical resolution to reconcile them. To address at least part of such questions, here we provide the maiden derivation of the static form of the de Sitter metric by solving the field equations.

In particular, here we focus attention to the question: "Why does the de Sitter metric appear to be perfectly static to an observer with respect to whom the cosmic fluid is expanding rapidly?"

\section{Results}

Maiden Derivation of the Static Form of de Sitter Metric by Solving Field Equations

In the Schwarzschild coordinate frame, one of the field equations is $^{3}$

$$
8 \pi T_{0}^{0}=-e^{-\lambda}\left(\frac{1}{R^{2}}-\frac{\lambda^{\prime}}{R}\right)+\frac{1}{R^{2}}
$$

where a prime denotes partial derivative by $R$. By integrating this equation, we obtain

$$
e^{-\lambda}=1-\frac{2 \mathcal{M}_{e}}{R}
$$

where

$$
\mathcal{M}_{e}=\int_{0}^{R} 4 \pi T_{0}^{0} R^{2} d R
$$


For the vacuum equation of state (EOS), $p_{e}=-\rho_{e}$, it is seen from Eq.(7) that

$$
T_{0}^{0}=\rho_{e}=\frac{\Lambda}{8 \pi}
$$

Thus we find that

$$
\mathcal{M}_{e}=\int_{0}^{R} 4 \pi \frac{\Lambda}{8 \pi} R^{2} d R=\frac{\Lambda R^{3}}{6}
$$

So from Eqs.(30) and (33), we promptly obtain

$$
e^{-\lambda}=1-\frac{\Lambda R^{2}}{3}=1-H^{2} R^{2}
$$

for the de Sitter metric. Another relevant field equation here is ${ }^{3}$

$$
8 \pi T_{1}^{1}=-e^{-\lambda}\left(\frac{1}{R^{2}}+\frac{\eta^{\prime}}{R}\right)+\frac{1}{R^{2}}
$$

Again for $p_{e}=-\rho_{e}$, we find from Eq.(8) that for the de Sitter case,

$$
T_{1}^{1}=-p_{e}=\frac{\Lambda}{8 \pi}
$$

Now by feeding equations (34) and (36) back into (35), it is found that

$$
\frac{2}{3}(\Lambda R)=-\eta^{\prime}\left(1-\Lambda R^{2} / 3\right)
$$

And this has a solution

$$
e^{\eta}=K\left(\frac{\Lambda R^{2}}{3}-1\right)
$$

Now, by demanding that, $e^{\eta}=1$ at $R=0$, we set the integration constant, $K=-1$, so that

$$
e^{\eta}=1-\frac{\Lambda R^{2}}{3}=1-H^{2} R^{2}
$$

Thus, by combining equations (7), (34) and (39), we derived here the de Sitter metric in the Schwarzschild frame, i.e., equations (20) and (21), by directly solving the relevant field equations without assuming $v=0$. And it is clear from this derivation, no EOS other than $p_{e}=$ $-\rho_{e}$ would have yielded such a static view of the metric.

\section{Discussions}

The de Sitter metric is a very important aspect of modern cosmology. It is also considered important for the present version of the superstring theory because it invokes the Anti- de Sitter Conformal Field Theory (AdS-CFT) correspondence. Essentially, it is conjectured that there is an equivalence between a string theory and gravity defined on one space, and a quantum field theory without gravity defined on the conformal boundary of this space, whose dimension is lower by one or more ${ }^{8,9}$.

Here we highlighted that there is a physical contradiction not only between the static and non-static versions of the de Sitter metric, but even between the old 1917 static interpretation and the present static interpretation. In 1917, the de Sitter static metric was supposed to describe a genuinely static background, $v(R, T)=0$, with a time independent energy density $\rho_{e}=\Lambda / 8 \pi$; in those days there was no concept of Raychoudhuri equation ${ }^{10}$ or expansion scalar ${ }^{1,2}$ or even a proper notion of a non-stationary metric. But following the developments of GR, and in particular FRW cosmology, the de Sitter metric is believed to be naturally non-static, and just a special form of the non-static FRW metric.

The appearance of the static Schwarzschild form of the de Sitter metric is considered as just a quirk coincidence. In particular, the entire question of the static or non-static view is ascribed to the peculiarites of the coordinate transformations relating the two forms:

$$
t=T+\frac{1}{H} \ln \sqrt{1-H^{2} R^{2}}
$$

and

$$
r=\frac{R}{\sqrt{1-H^{2} R^{2}}} e^{-H T} ; \quad \text { i.e., } R=r e^{H t}
$$

Historically, it was Lanczos ${ }^{11}$ who first pointed out that the static de Sitter metric can be given a non-static look by means of the above coordinate transformations. This work was however ignored in the literature and not mentioned in the comprehensive earlier books on GR like ${ }^{3,5,6}$. In any case, coordinate transformations obtained by mathematical trials and insights cannot offer physical explanation for staticity for a metric which appears to result in most rapid exponential time dependence in another frame.

But now, having solved for this original de Sitter metric, we can see that the static form is a result of the very special equation of state (EOS) of the vacuum fluid $p_{e}=-\rho_{e}$. With this EOS, the first two diagonal components of the effective energy momentum tensor remain the same in both the comoving and the noncomoving frames:

$$
T_{0}^{0}(\mathrm{com})=T_{0}^{0}(\text { ncom })=\frac{\Lambda}{8 \pi}
$$

and

$$
T_{1}^{1}(\mathrm{com})=T_{1}^{1}(\text { nom })=\frac{\Lambda}{8 \pi}
$$

Finally recall that in the non-comoving Schwarzschild frame, apparently, there should be a flow of mass energy even in the absence of heat/radiation flow because the fluid is in motion ${ }^{3}$. But with this special EOS, the non-comoving observer sees no mass-energy flux:

$$
T_{0}^{1}=\frac{\left(p_{e}+\rho_{e}\right) v e^{(\eta-\lambda) / 2}}{1-v^{2}}=0 ; \text { even if } v \neq 0
$$

Essentially the vacuum energy momentum tensor is Lorentz invariant and looks static to all observers. Many books and articles do mention that the de Sitter metric which is supposed to be non-static (in the comoving frame) appears to be static in the Schwarzschild frame. And there are of course very elaborate discussions on the geometry associated with de Sitter as well as anti de Sitter space-time geometries. But such discussions must not be confused with the explanation as to why precisely the exponentially expanding de Sitter metric can look static.

And now we highlight Energy Conservation Anomaly in the Two Frames:

We have already emphasized the fact, by definition, both the comoving and non-comoving de Sitter observer must measure the same value of the expansion scalar. But this assertion itself does not not eliminate the various other conflicts between the two observers discussed here. Note, though in GR one can use arbitrary coordinate systems and have different viewpoints, such as different observers being able to measure different values for the components of $T_{k}^{i}$, it is expected that the basic physics results should be unchanged. Thus indeed, various observers must not only measure the same values of physical scalars but should arrive at the same physical results. From such a viewpoint, one would expect all de Sitter observers to obey the "Principle of Energy Conservation" even though they are bound to measure different values of total energy; i.e., $E(\mathrm{com}) \neq E($ ncom $)$.

Before we start on this topic, first recall that, while the local energy momentum conservation in GR is ensured by $T_{b ; b}^{a}=0$, the question of global energy momentum conservation is a tricky one. One of the basic reasons for the ambiguity in even definiting a global energy is that the gravitational field can be made to vanish at any given spacetime point if one would use the local inertial frame. This problem is usually handled by recalling that, after all, there is no global inertial frame in the presence of mass-energy so that there should be a nonzero global total energy. Indeed, for an asymptotically flat spacetime, 
one can nicely define the "ADM Mass" as the global energy inclusive of matter and gravitation ${ }^{12}$. However if one would attempt to calculate the total energy using say an appropriate pseudo-tensor, it is seen that the calculated global energy depends on the coordinate system used. For example, if one would use Einstein's canonical pseudo-tensor, the total energy of a Minkowski vacuum would appear to blow up if spherical polar coordinates would be used. On the other hand, Cartesian coordinates indeed lead to zero total energy for the same spacetime. This only shows that energy measurements have meaning only with respect to a given "background reference spacetime" and probably Cartesian coordinates provide the most natural background. Thus starting from Einstein, all authors use the Cartesian coordinates for evaluating quatities involving pseudo-tensors ${ }^{13}$. Further, there could be many choices of pseudotensors and in certain cases, the global energy computed using different pseudo-tensors may differ. And such differences can arise for various quasilocal definitions of global energies too. It is found that "pseudotensor corresponds to a Hamiltonian boundary term. Hence, they are quasilocal and acceptable; each is the energy-momentum density for a definite physical situation with certain boundary conditions." ${ }^{\prime 4}$. Further, Einstein's canonical pseudo-tensor, calculated using Cartesian coordinates, appears to be highly physically significant because at least for an isolated object, the expression for total mass energy obtained using this pseudo-tensor matches with the corresponding Landau-Lifshitz expression ${ }^{3}$ (essentially the ADM mass) obtained without using any pseudo-tensor ${ }^{13}$.

To cut a long and involved story short, there is an unanimity that, at least for a spherically symmetric spacetime, global energy momentum is very much definable and Einstein's pseudotensor is very reliable. For a stationary system with timelike Killing vectors such a global energy must obviously be conserved. However, note that the question of non-conservation of global energy arises only for a system which has a boundary through which there could be incoming or outgoing flux of energy momentum. But, by definition, for the "universe", there is no external boundary. Thus for the special case of the universe, the global energy is expected to be conserved ${ }^{13}$. Accordingly, the global energy associated with the non-comoving de Sitter metric was reliably found to $b^{13}$

$$
E_{d S}(\mathrm{com})=\frac{\Lambda}{6} r^{3} a^{3}(t)=\frac{\Lambda}{6} r^{3} e^{\sqrt{3 \Lambda} t}
$$

To cross-check this, one can recall that, the total mass-energy of a spherically symmetric comoving fluid inclusive of gravitation is also given by the Misner-Sharp mass which is actually related to an invariant associated with the Riemann tensor $\mathcal{R}_{c d}^{a b}: M=\frac{1}{2} R^{3} \mathcal{R}_{\theta \phi}^{\theta \phi_{15}}$. Further the definition of Misner-Sharp mass coincides with that of several quasi-local energies and is the best measure of global energy for a spherically symmetric case ${ }^{16}$. With the inclusion of $\Lambda$, the expression for Misner-Sharp mass is

$$
M_{e}(r, t)=\int_{0}^{r} 4 \pi \rho_{e} R^{2}(\partial R / \partial r) d r
$$

And for the de Sitter case, with,

$$
R(r, t)=a(t) r
$$

one finds

$$
M_{e}(r, t)=\frac{\Lambda}{6} r^{3} a^{3}(t)=\frac{\Lambda}{6} r^{3} e^{\sqrt{3 \Lambda} t}
$$

It is interesting to see that we obtain the same expression for the total energy associated with the non-static de Sitter metric from two different and most reliable directions:

$$
E_{d S}(\operatorname{com})=M_{e}=\frac{\Lambda}{6} r^{3} e^{\Theta t}
$$

It is quite easy to physically verify the correctness of the above expression. The negative self-gravitational/binding energy of stars and other self-gravitating objects occur for systems with positive spatial curvature. And for the spatially flat de Sitter model, there is no negative self-gravitational energy. Even for supposed $k=+1,-1$ vacuum solutions, there should not be any negative self-gravitational energy because the vacuum cannot self-gravitate. Therefore, the global energy of the de Sitter spacetime solely arises from positive contributions of $\Lambda$. And since $\Lambda$ does not decrease with expansion/ contraction, one must have

$$
E_{d S}(\mathrm{com})=\frac{\Lambda}{8 \pi} \times V
$$

where $V$ is the proper spatial volume. Further, for spatially flat case, $V$ $=$ coordinate volume.

Therefore, clearly, from the perspective of the comoving frame, the principle of energy conservation is badly violated for the de Sitter universe if indeed $\Lambda \neq 0$.

In contrast, the static form of the de Sitter metric has a timelike Killing vector and obviously obeys this golden physical principle of energy conservation.

Thus the total energy of the de Sitter model increases in an exponential manner; such a bad violation of the "Principle of Conservation of Energy" in the comoving frame is in sharp contrast with the corresponding nice behaviour in the Schwarzschild frame.

To remind again, the expansion scalar $\Theta=\sqrt{3 \Lambda} \neq 0$ if indeed $\Lambda \neq$ 0 . Then, physically it is puzzling how can the de Sitter metric look static to any observer.

Indeed, at this moment, we have no answer to such pertinent questions. Prima-facie, all such conflicts would vanish if one would set the unknown ad-hoc constant $\Lambda=0$ in accordance with Einsteins's alleged self-proclaimation that the invention of a cosmological constant was his "biggest blunder". Also, in a future publication, we would attempt to address such issues. But, as of now, we have at least explained that the static form of the de Sitter metric is a result of very special vacuum $\operatorname{EOS} p=-\rho$.

One may also wonder, if indeed there is no mass energy flow for the vacuum fluid with respect to any observer, then how can physically this vacuum look to be exponentially expanding to some observer.

1. Grøn, $\varnothing$ \& Hetvik, S. Einstein's General Theory of Relativity With Modern Applications in Cosmology, (Springer, New York, 2007).

2. Krori, K. D. Fundamentals of Special \& General Relativity (PHI Learning, New Delhi, 2010).

3. Landau, L. \& Lifshitz, E. M. Classical Theory of Fields, (Pergamon, Oxford, 1962).

4. D'Inverno, R. Introducing Einstein's Relativity (Clarendon Press, Oxford, 2004).

5. Eddington, E. S. The Mathematical Theory of Relativity, (Cambridge Univ. Press, Cambridge, 1923).

6. Tolman, R. C. Relativity, Thermodynamics \& Cosmology, (Clarendon Press, Oxford, 1962).

7. de Sitter, W. On the relativity of inertia. Remarks concerning Einsteins latest hypothesis. Koninklijke Akademie van Wetenschappen te Amsterdam. Section of Sciences. Proceedings, 19, 1217-1225 (1917).

8. Maldacena, J. \& Strominger, A. Statistical entropy of de Sitter space. Journal of High Energy Physics 02, pp. 014 (1998).

9. Witten, E. Anti De Sitter Space And Holography. Adv. Theor. Math. Phys. 2, 253 (1998) http://arxiv.org/abs/hepth/9802150

10. Raychaudhuri, A. K. Relativistic cosmology I. Phys. Rev. 981123 (1955).

11. Lanczos, K. Bemerkung zur de Sitterschen Welt. Physikalische Zeitschrift, 23, 539-543 (1922).

12. Arnowitt R., Deser S. \& Misner, C. W. The dynamics of general relativity, in Witten, L., Gravitation: An Introduction to Current Research, Wiley, pp. 227-265 (1962).

13. Mitra, A. Einstein energy associated with the Friedmann-Robertson-Walker metric. Gen. Rel. and Grav. 42, 443-469 (2006); (arXiv:0911.2340).

14. Chang, C., Nester, J. M. \& Chen, C. Pseudotensors and Quasilocal EnergyMomentum. Phys. Rev. Letts. 83, 1897-1901 (1999).

15. Cahill, M. E. \& McVittie, G. C. Spherical Symmetry and Mass-Energy in General Relativity. I: General Theory. J. Math. Phys. 11, 1382-1391 (1970). 
16. Hayward, S. A. Gravitational Energy in Spherical Symmetry. Phys. Rev. D53, 1938-1949 (1996).

\section{Acknowledgements}

The author thanks the anonymous referee for raising a series of pertinent clarifications and making several helpful suggestions during three rounds of protracted revision. Further, he/ she has also helped in removing several typos and grammatical mistakes from the manuscript. The author is grateful to the referee for such constructive critiques.

\section{Additional information}

Competing financial interests The authors declare no competing financial interests.

License: This work is licensed under a Creative Commons

Attribution-NonCommercial-ShareAlike 3.0 Unported License. To view a copy of this license, visit http://creativecommons.org/licenses/by-nc-sa/3.0/

How to cite this article: Mitra, A. Interpretational conflicts between the static and non-static forms of the de Sitter metric. Sci. Rep. 2, 923; DOI:10.1038/srep00923 (2012). 